\title{
PML Effectiveness in the Transmission Line Modelling Method for Radiation and Scattering Applications
}

\author{
Jomiloju Odeyemi ${ }^{1}$, Chris Smartt ${ }^{1}$, Ana Vukovic ${ }^{1}$, Trevor M.Benson ${ }^{1}$ and Phillip Sewell ${ }^{1}$ \\ ${ }^{1}$ George Green Institute for Electromagnetics Research, University of Nottingham, Nottingham, NG7 2RD, UK \\ e-mail: Jomiloju.odeyemi@nottingham.ac.uk.
}

\begin{abstract}
This paper demonstrates the effectiveness of the recently introduced, stable, perfectly matched layer (PML) for the Transmission Line Modelling (TLM) method. The superiority of the new PML over the TLM matched boundary is demonstrated by application to electromagnetic scattering and radiation simulations.
\end{abstract}

Index Terms - Perfectly Matched Layer, stability, antennas, Transmission Line Modelling method, radar cross section.

\section{INTRODUCTION}

Since its development [1], the Transmission Line Modelling (TLM) method has been extensively applied to the study of numerous electromagnetic wave propagation problems. It offers an unconditionally stable algorithm by mapping Maxwell equations onto a set of equivalent transmission line equations. A circuit analogy is thus established which allows propagating fields to be modelled as voltage and current pulses propagating across an electrical network [1],[2].

Similar, to other differential equation based numerical methods, when applied to open boundary problems, e.g., in large scale radiation and scattering applications, the TLM method is required to absorb all outward going waves. Typically, this is achieved by either applying matched boundary conditions or introducing efficient absorbing boundary conditions (ABCs) at the edges of the computational domain.

Over the years, different ABCs have been proposed for terminating computational domains amongst which the perfectly matched layer (PML) is widely accepted as the preferred $\mathrm{ABC}$ for most practical applications [3],[4]. This is owed to its simplicity and the relatively high absorption performance attainable over all angles and frequencies of incidence. Numerous successful implementations of the PML have been demonstrated in the Finite Difference Time Domain (FDTD) method [4], however, the PML implementations in the TLM method are generally known to suffer some drawbacks.

The implementation of an effective PML in TLM is not trivial. Unlike the FDTD method, which supports a direct discretization of the PMLs equations, an effective TLM-PML formulation relies on deriving a suitable equivalent transmission line model. To date, the deployed PMLs in TLM demonstrate lower levels of absorption compared to those shown in FDTD simulations [4], [5]. In addition, until the recent TLM-PML developments introduced in [6], the unified PML implementations in TLM [5], [7] have generally been found to be numerically unstable and have therefore not been widely employed. For this reason, the simple TLM matched $\mathrm{ABC}[2]$, which terminates the computational domain with the wave impedance, is most commonly applied in practical applications. However, due to the matched ABCs poor absorption of oblique incidence waves the mesh boundaries must be placed at a significantly large distance from each radiating/scattering element in order to obtain accurate results thus degrading computational efficiency.

The PML formulation proposed in [6], which we refer to here as the mapped TLM-PML, demonstrated for the first time a stable unified TLM-PML implementation. The details of its implementation in three-dimensional (3D) setting is provided in [8] where its performance was evaluated for the canonical waveguide test case. The mapped TLM-PML performance for problems involving waves propagating outwardly in all directions such as scattering or antenna applications has yet to be reported. In these applications the PML must be applied at all edges of the problem domain which results in a PML overlap at the corner regions.

This paper demonstrates the effectiveness of the mapped TLM-PML in such radiation and scattering applications. Two test cases are studied.

The first test case involves the computation of the radar cross section (RCS) of a metal sphere illuminated by a plane wave and the second test case calculates the mutual impedance of two dipoles as a function of separation. Both test cases have been deliberately chosen as the results are sensitive to the performance of the boundary conditions applied to the truncated computational domain. The accuracy of the test cases is determined by benchmarking the results against the analytic solutions.

\section{FORMULATION}

A comprehensive description of the implementation of the mapped TLM-PML has been omitted here since this is provided in [8]. Nonetheless, the underlying equivalence which allows an implementation in the 3D TLM node is briefly provided in this section.

The stretched coordinate PML equation representing the $y$-projections of Ampere's law defined in a complex stretched space is given as [3] 


$$
\frac{1}{S_{z}} \frac{\partial H_{x}}{\partial z}-\frac{1}{S_{x}} \frac{\partial H_{z}}{\partial x}=\epsilon_{y} \frac{\partial E_{y}}{\partial t}+\sigma_{e y} E_{y}
$$

where $\mu_{y}$ and $\epsilon_{y}$ denote the permeability and permittivity, and $\sigma_{m y}$ and $\sigma_{e y}$ are the magnetic and electric conductivity of the medium and $S_{x}$, and $S_{z}$ are coordinate stretch factors in $x$ and $z$ directions which are compactly expressed as

$$
S_{i}=1+\frac{\sigma_{i}}{j \omega \epsilon_{0}}, \quad i=x, y, z
$$

where $\sigma_{i}$ represents the electric conductivity profile along the $i$-coordinate axis which controls the rate of decay. The coordinate stretch factors employed in (1) enable the desired attenuation of propagating waves and are defined according to stretched coordinate PML theory [3].

In its present form equation (1) does not reveal an equation suitable to establish a direct medium to circuit equivalence as given in [2]. However, a more suitable form can be obtained by multiplying both sides of (1) by $\frac{s_{z} s_{x}}{s_{y}}$. This yields a re-expression of (1) given as

$$
S_{x} \frac{\partial H_{x}}{\partial z}-S_{z} \frac{\partial H_{z}}{\partial x}=\frac{S_{z} S_{x}}{S_{y}} \epsilon_{y} \frac{S_{y} \partial E_{y}}{\partial t}+\frac{S_{z} S_{x}}{S_{y}} \sigma_{e y} E_{y} S_{y} .
$$

Upon close examination of (3) it can be seen that the medium parameters $\epsilon_{y}$ and $\sigma_{e y}$ are transformed by the stretch factor $\frac{s_{z} S_{x}}{s_{y}}$. According to the well-established TLM medium to circuit equivalence a relation between the node capacitance $\mathrm{C}$ and medium permittivity $\epsilon$, and node conductance $G$ and medium electric conductivity $\sigma_{e}$ exists. Therefore, the stretched coordinate PML theory can be implemented in TLM by a mapping of parameters such that $\epsilon \frac{S_{z} S_{x}}{S_{y}} \leftrightarrow C$ and $\frac{S_{z} S_{x}}{s_{y}} \sigma_{e} \leftrightarrow G$.

The mapped TLM-PML formulation is thus developed based on a mapping of the TLM node parameters to complex space which can also be physically interpreted as stretching the node about its centre (origin) from the real to a complex space.

\section{APPLICATIONS}

The mapped TLM-PML has been implemented in the open source TLM software GGI_TLM [9]. In GGI_TLM the PML is specified by its thickness $T H$, the theoretical reflection coefficient, $\mathrm{R}$, and the polynomial order of the conductivity profile, $\mathrm{n}$. The number of layers in the PML is given as $N=T H / \Delta l$, where $\Delta l$ is the TLM cell size. The electrical conductivity of the first layer of the PML, layer 0 is given by

$$
\sigma_{0}=\frac{\varepsilon_{0} c_{0} \ln R}{2 \Delta l N^{n+1}}
$$

and the conductivity of layer $L, 0 \leq L \leq N-1$ is given by

$$
\sigma_{L}=\sigma_{0}\left\{(L+1)^{n+1}-L^{n+1}\right\}
$$

In the GGI_TLM implementation the PML medium is situated inside the outer boundary and in the test cases presented in this paper the outer boundary voltage reflection coefficient is $\Gamma=0$ i.e. the PML is backed by the normal TLM matched boundary condition (although other reflection coefficients can be applied, this is found to be effective).

The effectiveness of the mapped TLM-PML boundary condition is demonstrated here in two test cases; a radar cross section calculation and the calculation of the mutual impedance of two antennas. In these simulations the performance of the $\mathrm{ABC}$ has a significant effect on the results. Comparisons between results with TLM matched boundary conditions at differing distances and PML will be presented. The test cases have analytic solutions and so reference results will also be presented.

The first test case consists of the calculation of the RCS of a perfectly conducting sphere. The sphere has a radius of $1 \mathrm{~m}$ and was positioned at the centre of the domain. It is illuminated by a pulse plane wave excited from a Huygens surface enclosing the sphere. The time domain scattered field is recoded on a second surface outside the Huygens surface and this is used to calculate the time domain far field and hence the frequency domain radar cross section. The mesh cell length is $\Delta l=0.025 \mathrm{~m}$ for all the simulation results presented. A typical mesh, showing the PML regions inside the outer boundary is shown in Fig.1. This mesh provides a sufficiently accurate result (with respect to mesh density) for the effects of the boundary conditions to be clearly observed.

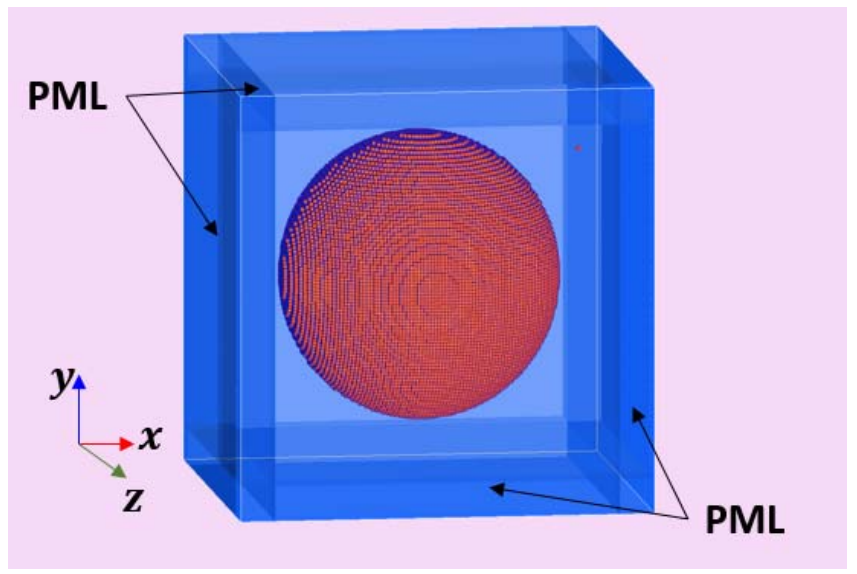

Fig. 1. GGI_TLM mesh for Sphere RCS calculation showing sphere and PML. The PML overlap at the corner regions are highlighted.

Four GGI_TLM simulations have been performed where the distance from the sphere's centre to the outer boundary were varied. For three test cases involving the matched boundary the distances were varied as follows: $1.5 \mathrm{~m}, 2.0 \mathrm{~m}$ and $4.0 \mathrm{~m}$. For the test case involving the PML a distance of $1.5 \mathrm{~m}$ was employed which included a PML medium which had a thickness of TH $=0.25 \mathrm{~m}$. The PML parameters were set as: $\mathrm{R}=10^{-4}$ and $\mathrm{n}=2$. 
The RCS results of the simulations conducted are shown along with the analytic solution [10] in Fig. 2. Clearly if a matched boundary is used (dashed lines in Fig. 2) then a distance of at least $4 \mathrm{~m}$ from the centre of the sphere to the outer boundary is required in order to obtain a reasonable result. However, a similar level of accuracy may be obtained using the PML boundary condition with a thickness of 10 cells $(T H=0.25 \mathrm{~m})$ in a mesh with outer boundary distance of only $1.5 \mathrm{~m}$.

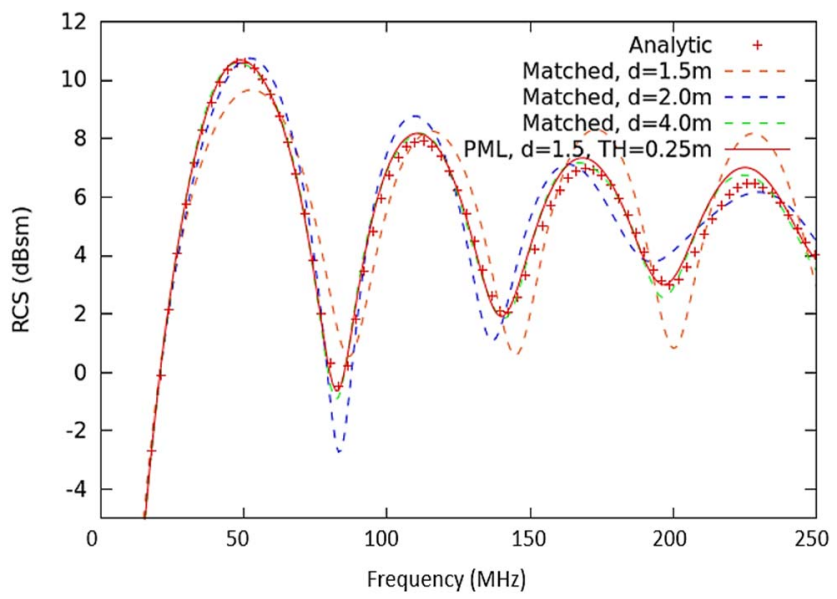

Fig. 2. RCS results of $1 \mathrm{~m}$ metal sphere obtained with matched boundaries (dashed curves), PML (solid curves) and analytic solution (crosses).

The second test case is the calculation of the coupling between two antennas. Here we calculate the coupling between two thin wire dipoles as a function of their separation using GGI_TLM. The $x$ directed dipoles are modelled using the TLM thin wire model [11]. The source dipole has a voltage source with a series resistance of $50 \Omega$ and the receiver dipole is loaded with a high impedance. In the simulation the time domain current on the source dipole, $i_{1}(t)$, and the voltage on the receiver dipole, $v_{2}(t)$, are recorded. These are Fourier transformed and the mutual impedance, $Z_{m}(f)=v_{2}(f) / i_{1}(f)$ is calculated.

The GGI_TLM mesh for one separation is shown in Fig. 3 where both dipoles are $0.05 \mathrm{~m}$ long and the dipole wire radii are approximately $0.01 \mathrm{~mm}$. The mutual impedance for separations in the $\mathrm{z}$ direction from $0.01 \mathrm{~m}$ to $1 \mathrm{~m}$ are calculated at a frequency of $2.7 \mathrm{GHz}$ where the distance to the outer boundary in the $x$ and $y$ directions is kept constant. Each separation requires a different mesh and a separate simulation. This is a testing problem for absorbing boundary conditions as the aspect ratio of the meshes become very large for large antenna separations and reflections from the outer boundaries at close to grazing angles become significant.

Six GGI_TLM simulations have been performed in the dipole mutual impedance tests. Four test cases involved employing the matched boundary where the distance to the outer boundary were varied as follows: $0.0525 \mathrm{~m}, 0.1025 \mathrm{~m}$, $0.1525 \mathrm{~m}$, and $0.2025 \mathrm{~m}$. A PML with a thickness of $\mathrm{TH}=0.025 \mathrm{~m}$ was employed in cases with distances of
$0.0525 \mathrm{~m}$ and $0.1025 \mathrm{~m}$. The PML parameters were set as: $\mathrm{R}=10^{-4}$ and $\mathrm{n}=2$.

The results of these simulations are shown in Fig.4 which shows $\left|Z_{m}\right|$ in $\mathrm{dB}$ i.e. the magnitude of the mutual impedance along with the results of the analytic solution to this problem [11]. The results using the matched boundary (dashed lines in Fig. 4 ), whilst being reasonable for separations up to $0.01 \mathrm{~m}$, show significant deviations from the expected results for higher separations due to reflections from the outer boundary of the problem space. The two PML results (solid lines in Fig. 4) are very close to the analytic result, even for very large separations, though some small deviation is visible. Note that the same PML parameter specifications have been used for all of the dipole separation simulations and no attempt has been made here to optimise the PML for each particular case.

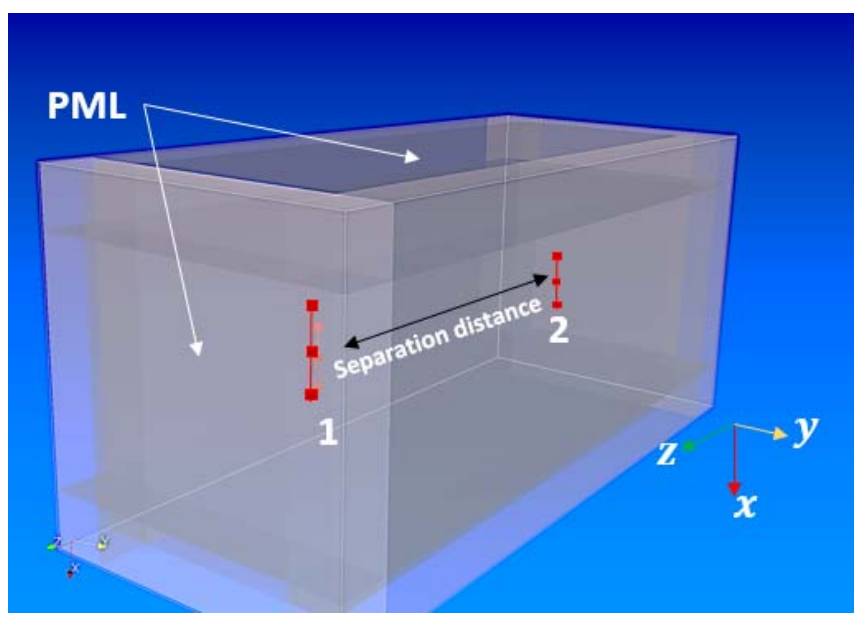

Fig. 3. GGI_TLM mesh for the dipole coupling model (including PML). The source dipole is labelled as " 1 " and the receiver dipole is labelled as " 2 ".

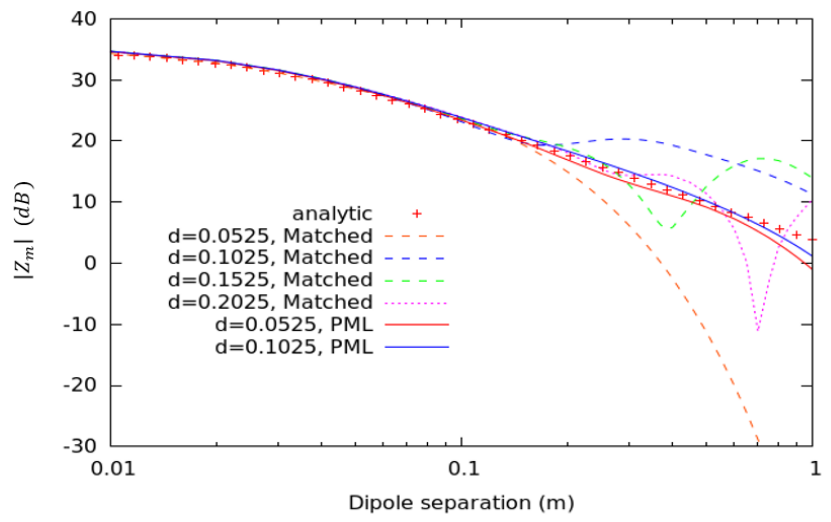

Fig. 4. Magnitude of mutual impedance as a function of distance. GGI_TLM results with matched boundaries (dashed curves), PML (solid curves) and analytic solution (crosses).

Fig. 5 and Fig. 6 show the magnitude of the $E_{x}$ field component at $2.7 \mathrm{GHz}$ over the problem space for a dipole separation of $0.04 \mathrm{~m}$ for the matched boundary and PML respectively. Both simulations are for an outer boundary distance of $0.1525 \mathrm{~m}$. 
The matched boundary field plot in Fig. 5 clearly shows the interference effect of the field reflected from the outer boundary whereas the PML field plot in Fig. 6 shows the absorption of the outward going wave in the PML and the expected undistorted spherical waves in the far field of the source dipole antenna. The matched boundary field plot shows a very low field value in the region of the receiving antenna; this corresponds to the minimum in the green dashed curve in Fig. 4.

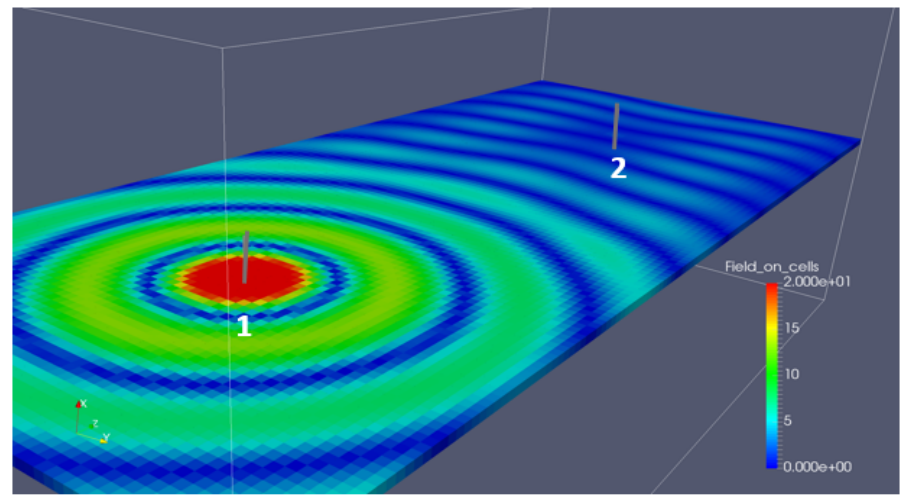

Fig. 5. Magnitude of the $\mathrm{E}_{\mathrm{x}}$ field component at $2.7 \mathrm{GHz}$ with a matched boundary for a dipole separation of $0.04 \mathrm{~m}$.

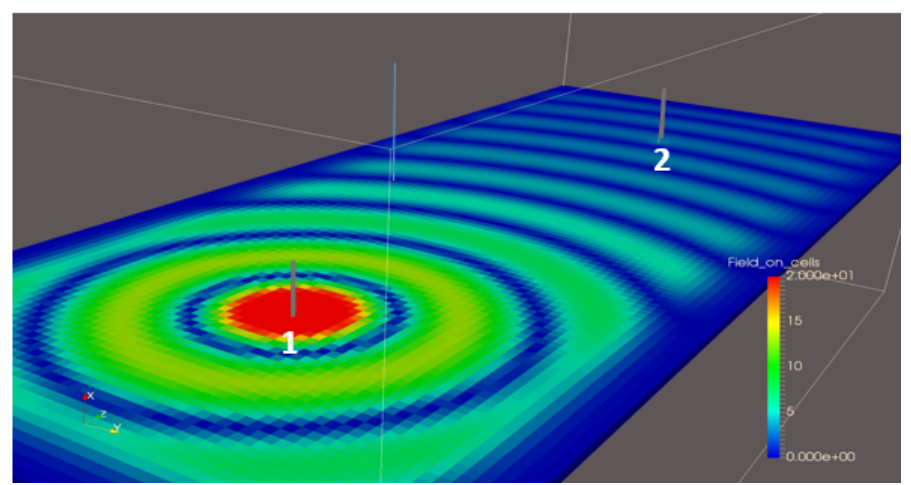

Fig. 6. Magnitude of the $\mathrm{E}_{\mathrm{x}}$ field component at $2.7 \mathrm{GHz}$ with a PML for a dipole separation of $0.04 \mathrm{~m}$.

\section{CONCLUSIONS}

The PML are considered to be state of the art ABC and when optimized significant improvements in the computational efficiency can be achieved. In this paper the effectiveness of the new PML implementation in the TLM algorithm has been demonstrated by application to $3 \mathrm{D}$ scattering and radiation test cases. Comparison of the PML results with those of the standard TLM matched boundary have shown a very significant improvement in the accuracy of the results is obtained even when the PML medium is placed very close to the radiating/scattering object. In addition the PML simulations have not shown any signs of numerical instability, thus we conclude that this is a highly effective absorbing boundary condition for TLM. The PML algorithm is implemented in the open source software, GGI_TLM.

\section{REFERENCES}

[1] P.B. Johns and R.L. Beurle, "Numerical Solution of 2- Dimensional Scattering Problems using a Transmission Line Matrix" Pruc. IEE, vo1.118, no. 9, pp 1203-1208, Sept. 1971

[2] C. Christopoulos, The Transmission-Line Modeling Method: TLM. IEE Press: Piscataway, NJ, 1995

[3] W.C. Chew and W.H. Weedon, "A 3D perfectly matched medium from modified Maxwell's equations with stretched coordinates," Microwave and Optical Tech. Lett., vol. 7, no. 13, pp. 599-604, 1994

[4] J.P. Bérenger,"Perfectly matched layer (PML) for computational electromagnetics. Synthesis lectures on computational electromagnetics," Morgan and Claypool, 2007

[5] J.L. Dubard and D. Pompei, "Optimization of the PML efficiency in 3D TLM method." IEEE Transactions on Microwave Theory and Techniques; 48(7):1081-1087, 2000

[6] J. Odeyemi, A. Vukovic, T. Benson, P. Sewell, "An improved PML implementation in the transmission line method." IET 10th International Conference on Computational Electromagnetics, June 2019

[7] L. Maguer, and M.M. Ney, "Extended PML-TLM node: an efficient approach for fullwave analysis of open structures." Int. J. Numer. Model., vol. 14 , pp. 129-144, 2001

[8] J. Odeyemi, A. Vukovic, T. Benson and P. Sewell, "A complex domain mapping of the SCN for an effective PML implementation in TLM. (Unpublished)

[9] GGI_TLM open source TLM software https://github.com/ggiemr/GGI_TLM

[10] G.T. Ruck, D.E. Barrick, W D. Stuart and C.K. Krichbaum, "Radar Cross Section Handbook," vol 1, 1970, Plenum Publishing Co

[11] A.J. Wlodarczyk, V. Trenkic, R.A. Scaramuzza and C. Christopoulos, "A fully integrated multiconductor model for TLM," IEEE Trans. MTT, vol. 46, no 2, pp 2431-2437, Dec 1998

[12] G.H. Brown and R. King, "High Frequency Models in Antenna Investigations," Proc IRE, vol 22, pp 457-480, 1934 